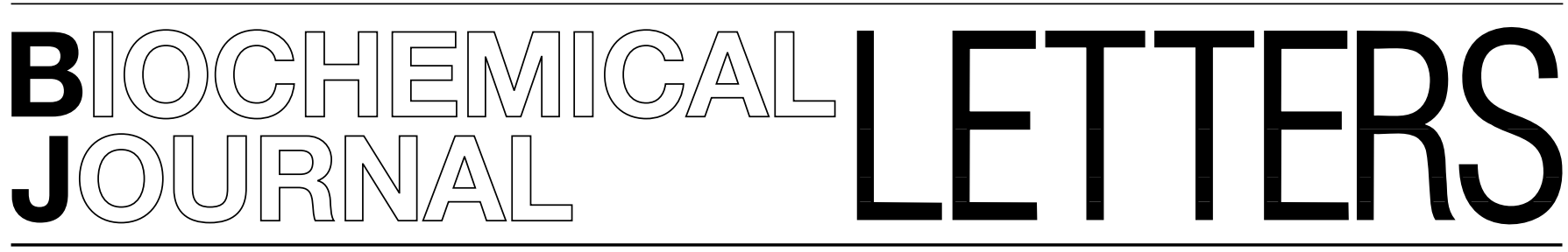

\section{A classification of nucleotide-diphospho-sugar glycosyltransferases based on amino acid sequence similarities}

Glycosyl-transfer reactions are, on quantitative terms, the most important biotransformations on Earth, since they account for the biosynthesis and hydrolysis of the bulk of biomass [1]. The biosynthesis of polysaccharides and complex carbohydrates is also of fundamental biological importance, since these molecules of fascinating diversity directly mediate a wide range of functions, from structure and storage to specific signalling. The biosynthesis of disaccharides, oligosaccharides and polysaccharides involves the action of hundreds of different glycosyltransferases (EC 2.4.x.y), enzymes which catalyse the transfer of sugar moieties from activated donor molecules to specific acceptor molecules, forming glycosidic bonds. There is a parallel extensive diversity of glycoside hydrolases (EC 3.2.1.x), enzymes which cleave such bonds to yield carbohydrates smaller than those from whence they originated. The immense functional and structural variety of glycosyltransferases and glycoside hydrolases raises the problem of their classification.

Regardless of the direction of the reaction, enzymes which catalyse glycosyl-transfer reactions can be classified according to the stereochemistries of the reaction substrates and products as either ' retaining' or 'inverting' enzymes [2]. Furthermore, specific enzymes can be classified on the basis of the reaction catalysed and the substrate specificity, according to the recommendations of the International Union of Biochemistry and Molecular Biology (IUBMB) [3]. However, there are limitations to the utility of this system for classification of glycosyltransferases and glycoside hydrolases, as it does not indicate the intrinsic structural features of the enzymes, nor does it adequately accommodate enzymes which act on several distinct substrates.

Classification of enzymes based on the similarities of their amino acid sequences offers a system complementary to that of the IUBMB [3] and realizes the potential to marry structural features of enzymes with their observed functions. Such classification systems have been proposed for glycoside hydrolases [4] and peptidases [5], and have been updated with the increasing number of cloned genes for these enzymes [6-9]. A significant advantage of classification according to sequence similarities is that it allows logical grouping of enzymes of different EC numbers into polyspecific families and offers insights into the divergent evolution of enzyme families [4]. Conversely, some enzymes which can be grouped by function have been shown to belong to several distinct families and thus reflect convergent evolution [4]. Significantly, the discriminatory power of these classifications has been confirmed by the similarity of the threedimensional structures [10] and the conserved molecular mechanisms [11] of family members.

Despite the utility of the sequence-based classification of glycoside hydrolases, no such system has been fully described for glycosyltransferases. One difficulty with such a classification is the number of enzymes concerned (193 entries of EC 2.4.1.x), and the diversity of sugar donors. These can be di- or poly- saccharides, sugar 1-phosphates, or, most commonly, nucleotide diphospho-sugars (NDP-sugars). Whereas a number of the latter type of glycosyltransferases have been compared and grouped into a single family [12], and sequence similarity has been used to predict mechanisms of action [13], there have been no reports of a comprehensive classification of NDP-sugar glycosyltransferases. The present letter describes a classification of NDP-sugar hexosyltransferases (EC 2.4.1.x) and related proteins into distinct sequence-based families.

Sequences of NDP-sugar hexosyltransferases were retrieved from the SwissProt and EMBL/GenBank databanks and compiled into a preliminary sequence library which covered the 35 EC 2.4.1.x entries for which at least one sequence is known to date. Representatives of each EC number were used as templates for BLAST similarity searches [14], and complementary sequences were retrieved from either SwissProt or EMBL/GenBank. BLAST results were examined using Visual BLAST [15]. When the BLAST probability values were low (typically $P>$ $10^{-3}$ ), sequences were further compared by hydrophobic cluster analysis (HCA) [16,17]. A family was defined as a grouping of at least two sequences of significant amino acid or HCA similarity over a length exceeding 100 residues, with no similarity to other families.

A total of 555 sequences were analysed, of which 553 were classified into 26 families (Table 1). Only two sequences, namely those of the mannosyltransferase OCH1 of Saccharomyces cerevisiae (GenBank D11095) and the DNA $\beta$-glucosyltransferase of bacteriophage T4 (Swiss-Prot P04547), could not be assigned to any family and were left unclassified.

Seven families were found polyspecific (containing two or more EC numbers), whereas the others were either monospecific (one single EC number) or 'uncertain' (no EC numbers assigned to the sequences). Previous experience with the classification of glycoside hydrolases suggests that the number of polyspecific families could increase with the availability of more glycosyltransferase sequences.

More than half of the sequences are found in the three largest families (families 1, 2 and 4 with respectively 107, 139 and 84 members; Table 1). Family 1 comprises proteins from viruses, bacteria, fungi, plants and animals. Families 2, 4, 8 and 20 contain sequences from bacteria, fungi, plants and animals. Conversely, several other small families appear strongly biased toward only one taxonomic group, but this could simply be a consequence of the smaller number of current members in these families.

Sequence similarity is strongly indicative of folding similarity in proteins [18]. Conservation of tertiary structure is such that the same three-dimensional fold is expected to be found within each of the families defined by the present study. For polyspecific families, this suggests that details of the three-dimensional structure, rather than differences in the global fold, will explain different donor and/or acceptor specificities. Whereas to date there has been only one reported three-dimensional structure for a glycosyltransferase, the DNA $\beta$-glucosyltransferase of bacteriophage T4 [19], it is inevitable that more of these enzymes will be purified, crystallized and characterized. Family allocation 


\section{Table 1 Families of NDP-sugar hexosyltransferases and related protein sequences}

The mechanism (retaining or inverting) is indicated for each family where it could be unambiguously identified from the sequence databanks or from the EC recommendations. Notes: ${ }^{(a)}$ Description as found in the sequence databanks; ${ }^{(b)} \mathrm{EC}$ number as found in the sequence databanks; ${ }^{(c)}$ accession numbers starting with $\mathrm{P}$ or $\mathrm{Q}$ are from the Swiss-Prot databank, those starting with $\mathrm{PC}$ are from the PIR databank and those starting with other letters are from the EMBL/GenBank databanks. For conciseness, the names of several enzymes have been abbreviated when a known EC number could be given. Specifically, this has been done for EC 2.4.1.101 ( $\beta$-1,3-mannosyl-glycoprotein $\beta$-1,2-N-acetylglucosaminyltransferase), EC 2.4.1.102 ( $\beta$-1,3-galactosyl- 0 -glycosyl-glycoprotein $\beta$ 1,6- $\mathrm{N}$-acetylglucosaminyltransferase), EC 2.4.1.143 ( $\alpha$-1,6-mannosyl-glycoprotein $\beta$-1,2-N-acetylglucosaminyltransferase), EC 2.4.1.144 ( $\beta$-1,4-mannosyl-glycoprotein $\beta$-1,4- $N$-acetylglucosaminyltransferase), and EC 2.4.1.155 [ $\alpha-1,3(6)$-mannosyl-glycoprotein $\beta$-1,6-N-acetylglucosaminyltransferase]. Abbreviations used: GPI, glycosyl-phosphatidylinositol; LPS, lipopolysaccharide; GlcNAC, N-acetylglucosamine.

Description $^{(a)} \quad$ EC number ${ }^{(b)} \quad$ Organism Accession no. ${ }^{(c)}$

Family 1 (inverting)

Glycosyltransferase GtfA

Glycosyltransferase GttB

Glycosyltransferase GitC

Glycosyltransferase GitfD

Glycosyltransferase Gitte

Ecdysteroid glucosyltransferase

Unknown AC3.2

Unknown AC3.7

Unknown AC3.8

Unknown B0310.5

Unknown C07A9.6

Unknown C08B6.1

Unknown C17G1.3

Unknown C18C4.3

Unknown C23G10.6

Unknown C33A12.6

Unknown C35A5.2

Unknown C44H9.1

Unknown C55H1.1

Unknown F01E11.1

Unknown F08G5.5

Unknown F29F11.2

Unknown F35H8.6

Unknown R04B5.9

Unknown R11A8.3

Unknown T04H1.7

Unknown T04H1.8

Unknown T07C5.1

Unknown T25B9.7

Unknown ZC443.6

Unknown ZC455.3

Unknown ZC455.4

Unknown ZC455.5

Unknown ZC455.6

Ecdysteroid glucosyltransferase

Ecdysteroid glucosyltransferase

CrtX protein

Zeaxanthin glucosyltransferase

Zeaxanthin glucosyltransferase

Flavonol $O^{3}$-glucosyltransferase

Flavonol $O^{3}$-glucosyltransferase

2.4.1.91

2.4.1.91

1 - $\beta$-Galactosyltransferase

Glucuronosyltransferase 1B

Glucuronosyltransferase $1 \mathrm{C}$

Glucuronosyltransferase $1 \mathrm{D}$

Glucuronosyltransferase $1 \mathrm{E}$

Glucuronosyltransferase $1 \mathrm{~A}$

Glucuronosyltransferase $1 \mathrm{~F}$

Glucuronosyltransferase 2B10

Glucuronosyltransferase 2B11

Glucuronosyltransferase 2B4

Glucuronosyltransferase 2B7

Glucuronosyltransferase 2B8

Glucuronosyltransferase 2B15

Ecdysteroid glucosyltransferase

Twi1 protein

Ecdysteroid glucosyltransferase

Ecdysteroid glucosyltransferase

UDP-glucose glucosyltransferase Cgt1

UDP-glucose glucosyltransferase Cgt5

Galactosyltransferase

Glucuronosyltransferase 1A1

\begin{tabular}{|c|c|c|}
\hline & Amycolatopsis orientalis & U84349 \\
\hline & Amycolatopsis orientalis & U84349 \\
\hline & Amycolatopsis orientalis & U84349 \\
\hline & Amycolatopsis orientalis & U84350 \\
\hline & Amycolatopsis orientalis & U84350 \\
\hline & Autographa californica NP virus & P18569 \\
\hline & Caenorhabditis elegans & Z71177 \\
\hline & Caenorhabditis elegans & Z71177 \\
\hline & Caenorhabditis elegans & Z71177 \\
\hline & Caenorhabditis elegans & U40959 \\
\hline & Caenorhabditis elegans & P34317 \\
\hline & Caenorhabditis elegans & $\mathrm{Z} 72502$ \\
\hline & Caenorhabditis elegans & Z78415 \\
\hline & Caenorhabditis elegans & U55369 \\
\hline & Caenorhabditis elegans & U39851 \\
\hline & Caenorhabditis elegans & Z68493 \\
\hline & Caenorhabditis elegans & Z71185 \\
\hline & Caenorhabditis elegans & $Z 75529$ \\
\hline & Caenorhabditis elegans & U55367 \\
\hline & Caenorhabditis elegans & U42832 \\
\hline & Caenorhabditis elegans & $\mathrm{Z70682}$ \\
\hline & Caenorhabditis elegans & Z73905 \\
\hline & Caenorhabditis elegans & Z36752 \\
\hline & Caenorhabditis elegans & $\mathrm{Z} 70782$ \\
\hline & Caenorhabditis elegans & Z70310 \\
\hline & Caenorhabditis elegans & $\mathrm{Z78200}$ \\
\hline & Caenorhabditis elegans & Z78200 \\
\hline & Caenorhabditis elegans & Z50006 \\
\hline & Caenorhabditis elegans & Z70311 \\
\hline & Caenorhabditis elegans & Z75553 \\
\hline & Caenorhabditis elegans & Z75554 \\
\hline & Caenorhabditis elegans & Z75554 \\
\hline & Caenorhabditis elegans & $\mathrm{Z75554}$ \\
\hline & Caenorhabditis elegans & 275554 \\
\hline & Choristoneura fumiferana NP virus 1 & U10441 \\
\hline & Choristoneura fumiferana NP virus 2 & U10476 \\
\hline & Erwinia herbicola & M90698 \\
\hline & Erwinia herbicola & Q01330 \\
\hline & Erwinia uredovora & P21686 \\
\hline 2.4.1.91 & Gentiana triflora & D85186 \\
\hline 2.4.1.91 & Hordeum vulgare & P14726 \\
\hline 2.4.1.45 & Human & U32370 \\
\hline 2.4.1.17 & Human & P36509 \\
\hline 2.4.1.17 & Human & P35503 \\
\hline 2.4.1.17 & Human & P22310 \\
\hline 2.4.1.17 & Human & P35504 \\
\hline 2.4.1.17 & Human & P22309 \\
\hline 2.4.1.17 & Human & P19224 \\
\hline 2.4.1.17 & Human & P36537 \\
\hline 2.4.1.17 & Human & P36538 \\
\hline 2.4.1.17 & Human & P06133 \\
\hline 2.4.1.17 & Human & P16662 \\
\hline 2.4.1.17 & Human & P23765 \\
\hline 2.4.1.17 & Human & P54855 \\
\hline & Lacanobia oleracea granulosis virus & Y08294 \\
\hline & Lycopersicon esculentum & X85138 \\
\hline & Lymantria dispar multicapsid NP virus & P41713 \\
\hline & Mamestra brassicae NP virus & U41999 \\
\hline & Manihot esculenta & X77459 \\
\hline & Manihot esculenta & X77462 \\
\hline 2.4.1.62 & Mouse & X92122 \\
\hline 2.4.1.17 & Mouse & D87867 \\
\hline
\end{tabular}

U84349

U84350

884350

Z71177

40959

2502

3985

771185

275529

42832

770682
773905

Z36752

8200

270006

270311

27555

775554

Z75554

M90698

D85186

P14726

P36509

P35503

P35504

P22309

P19224

P36538

23765

Y88294

$\times 85138$

U41999

$X 77459$
$\times 77462$

D87867 
Table 1 (cont.)

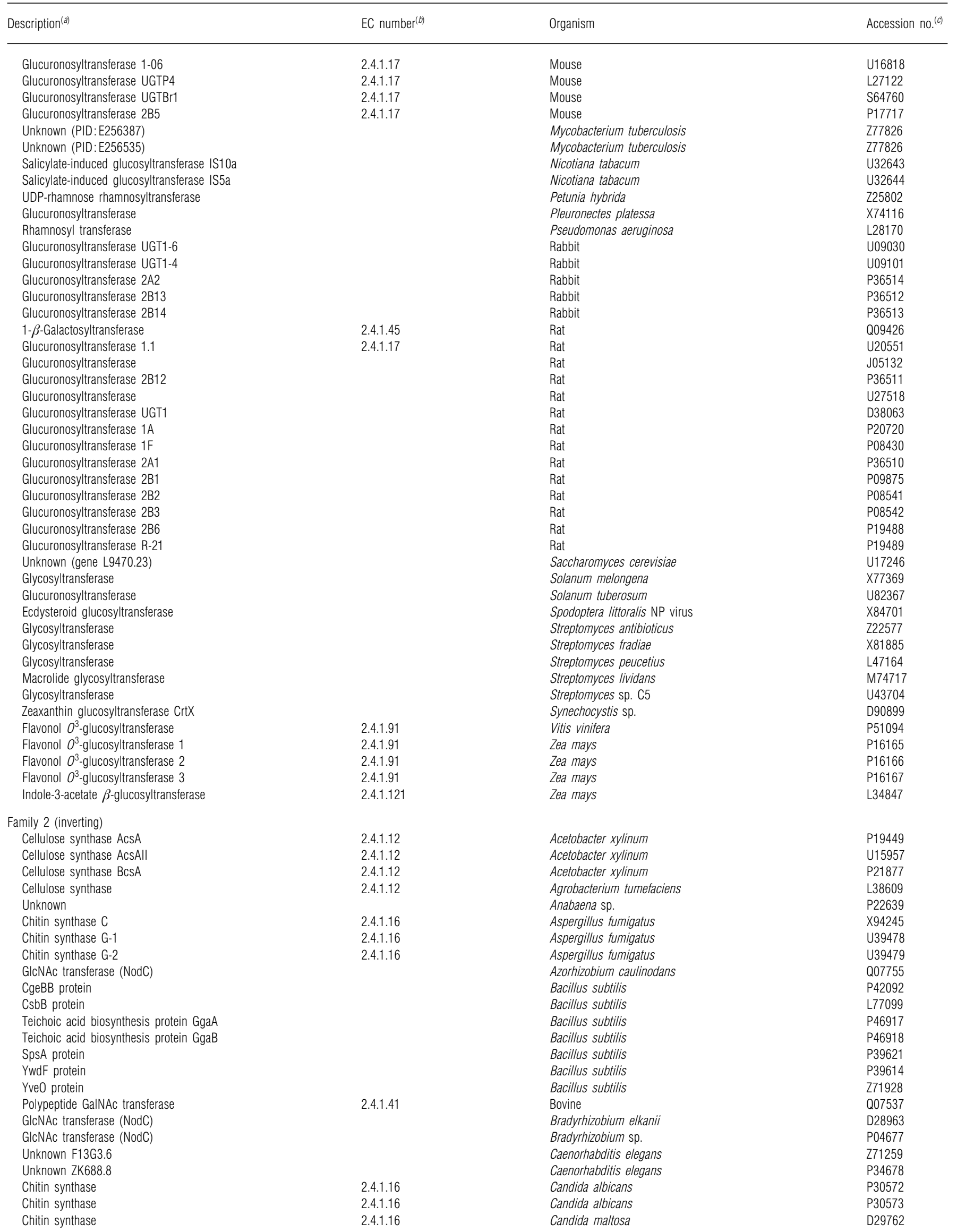


Table 1 (cont.)

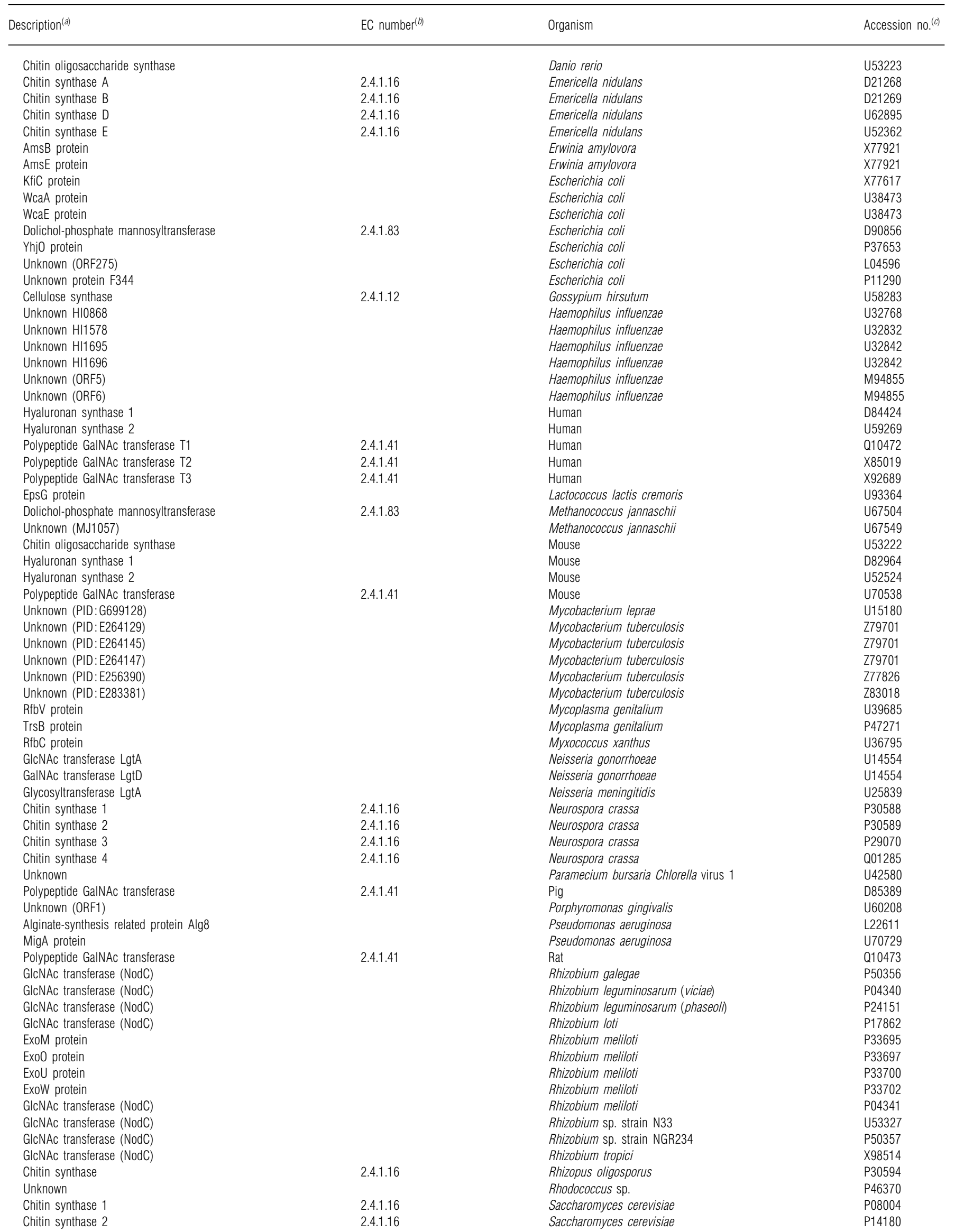


Table 1 (cont.)

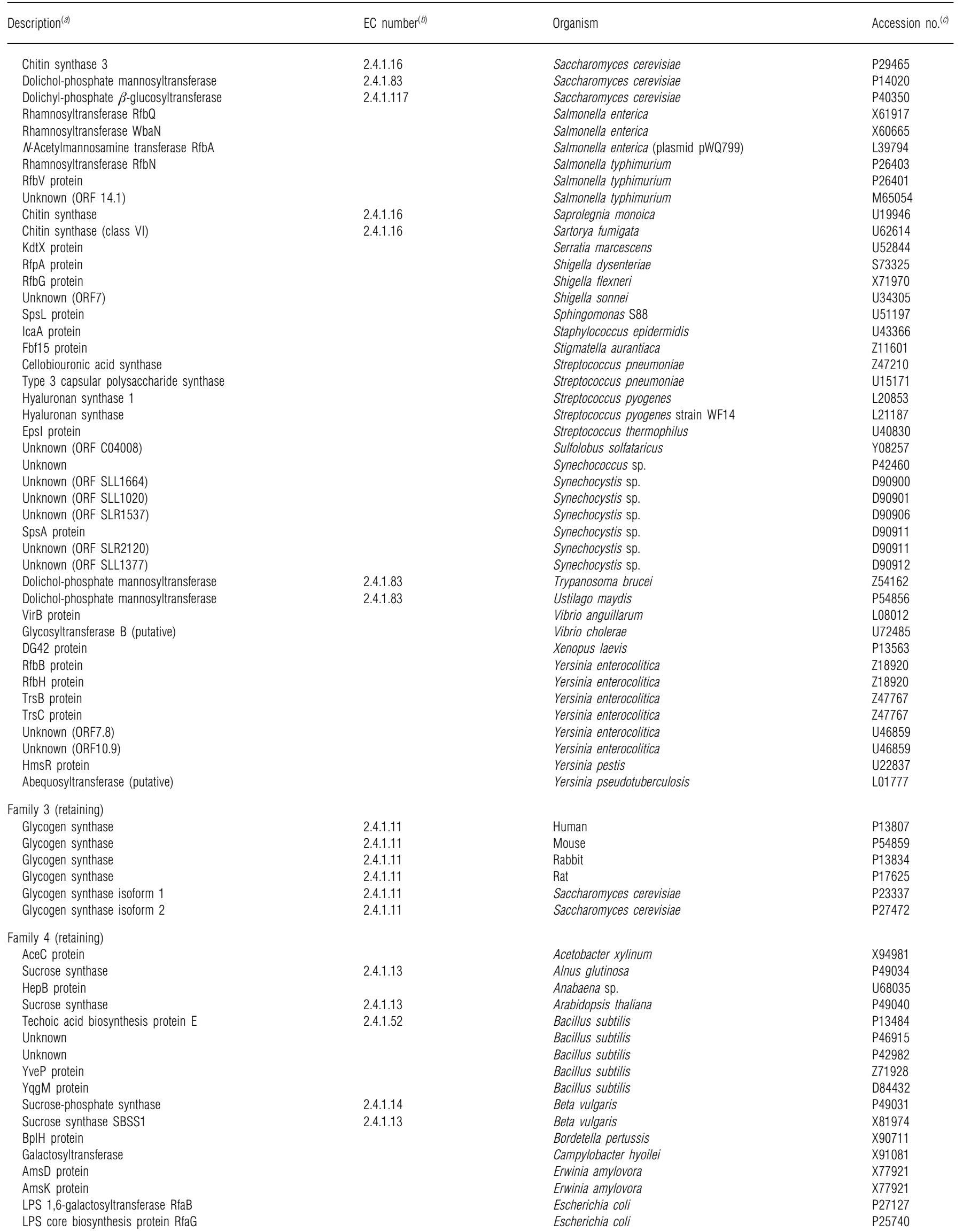


Table 1 (cont.)

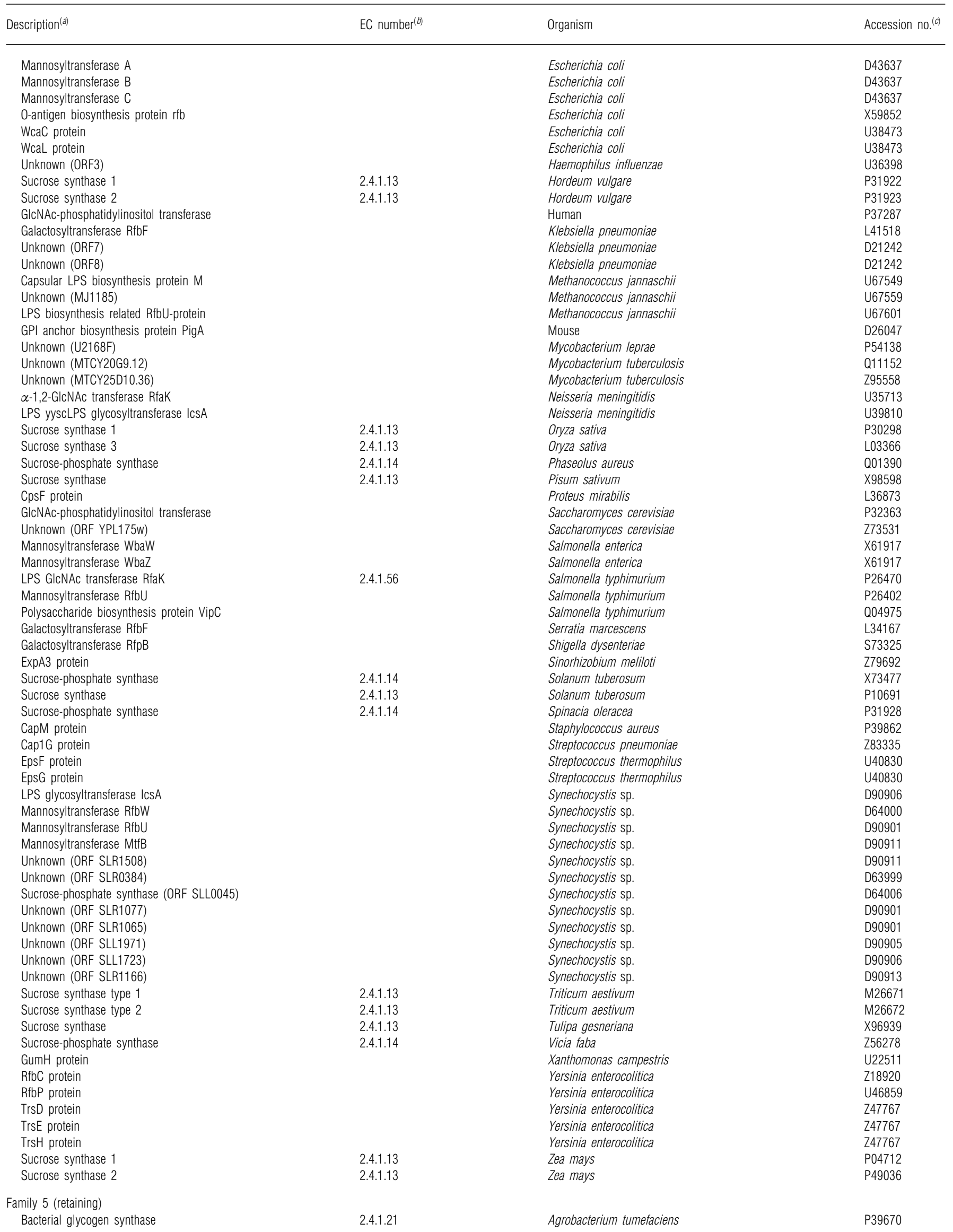


Table 1 (cont.)

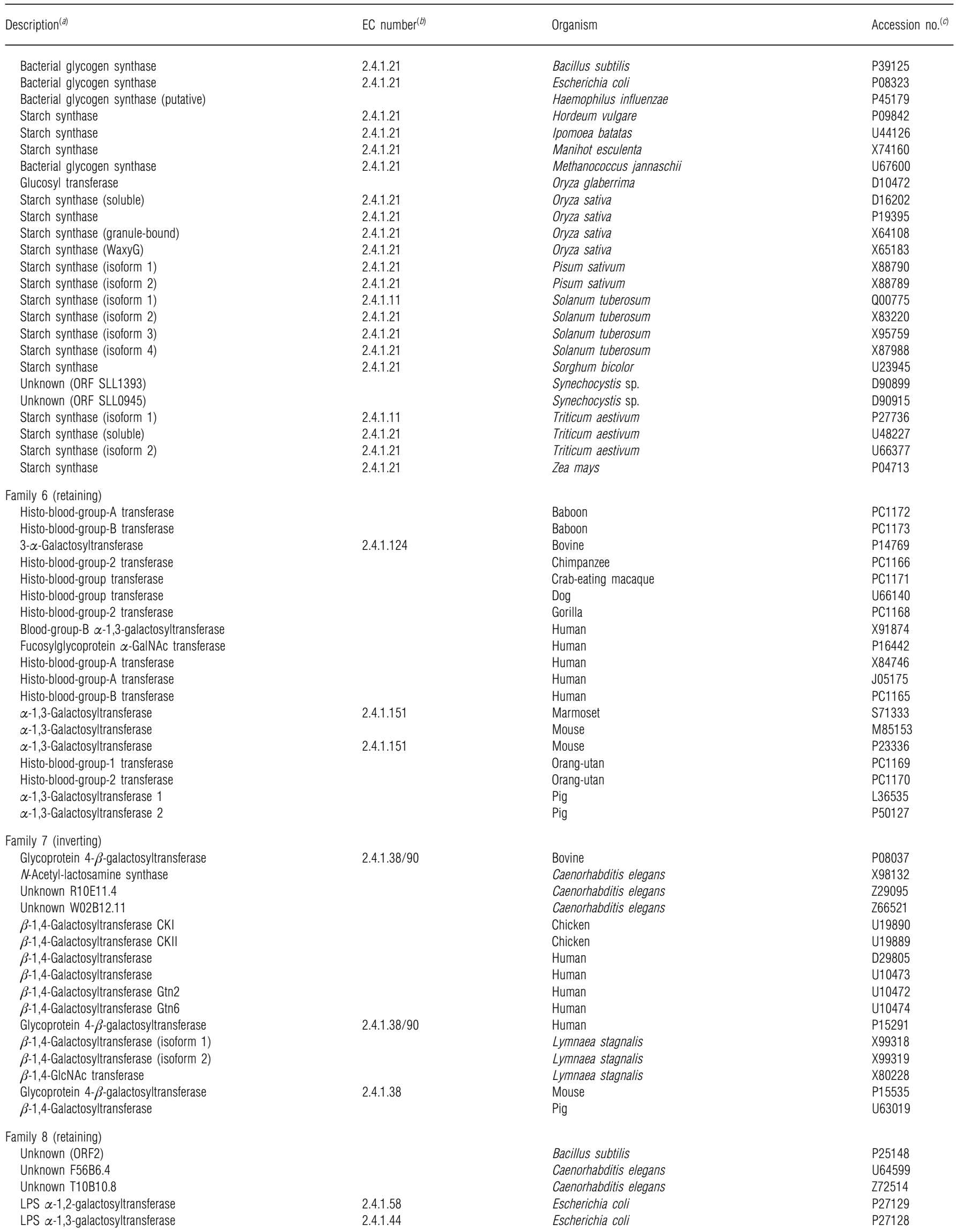


Table 1 (cont.)

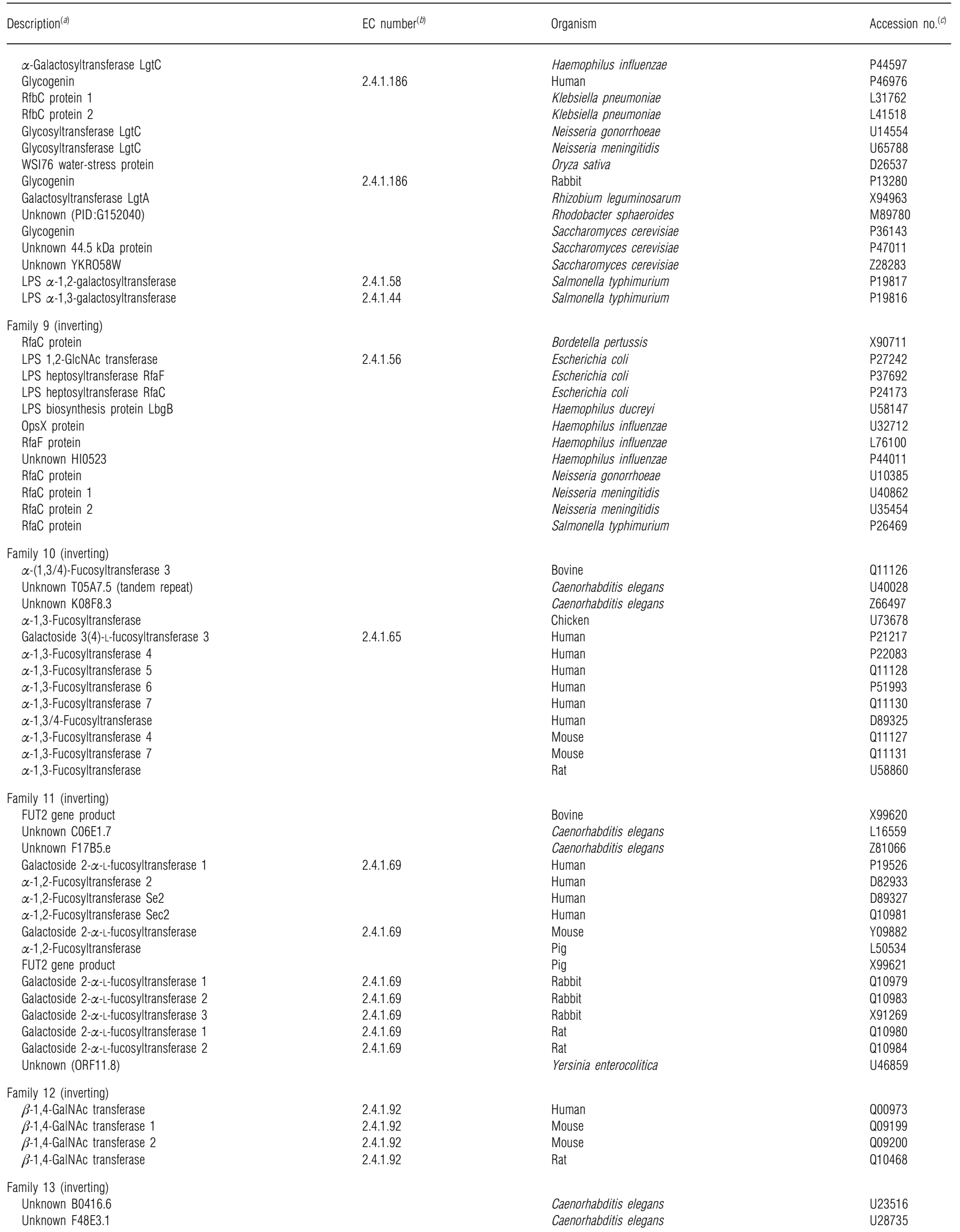


Table 1 (cont.)

\begin{tabular}{|c|c|c|c|}
\hline Description $^{(a)}$ & EC number ${ }^{(b)}$ & Organism & Accession no. ${ }^{(c)}$ \\
\hline Unknown M01F1.1 & & Caenorhabditis elegans & Z46381 \\
\hline GlcNAc transferase I & & Cricetulus griseus & U65791 \\
\hline$\beta-1,2-G \mid c N A c$ transferase I & 2.4.1.101 & Mouse & P27808 \\
\hline$\beta$-1,2-GIcNAc transferase I & 2.4.1.101 & Rabbit & P27115 \\
\hline$\beta$-1,2-GlcNAc transferase I & 2.4.1.101 & Rat & Q09325 \\
\hline Unknown T14B4.9 & & Caenorhabditis elegans & U50191 \\
\hline Unknown F22D6.11 & & Caenorhabditis elegans & Z71262 \\
\hline Unknown F30A10.4 & & Caenorhabditis elegans & Z81072 \\
\hline Unknown F44F4.6 & & Caenorhabditis elegans & Z37092 \\
\hline Unknown R07B7.6 & & Caenorhabditis elegans & 275955 \\
\hline Unknown T09E11.a & & Caenorhabditis elegans & 281147 \\
\hline$\beta$-1,6-GICNAc transferase C2GNT & 2.4.1.102 & Human & Q02742 \\
\hline \multicolumn{4}{|l|}{ Family 15 (retaining) } \\
\hline Mannosyltransferase Mnt1 & 2.4.1.131 & Candida albicans & X99619 \\
\hline Mannosyltransferase Mnt2 & 2.4.1.131 & Candida albicans & P46592 \\
\hline Glycolipid 2- $\alpha$-mannosyltransferase & 2.4.1.131 & Saccharomyces cerevisiae & P27809 \\
\hline Mannosyltransferase Ktr1 & 2.4.1.131 & Saccharomyces cerevisiae & P27810 \\
\hline Mannosyltransferase Ktr2 & 2.4.1.131 & Saccharomyces cerevisiae & P33550 \\
\hline Mannosyltransferase Ktr3 & 2.4.1.131 & Saccharomyces cerevisiae & P38130 \\
\hline Mannosyltransferase Ktr4 & 2.4.1.131 & Saccharomyces cerevisiae & P38131 \\
\hline Mannosyltransferase Ktr5 & 2.4.1.131 & Saccharomyces cerevisiae & P53966 \\
\hline Mannosyltransferase Ktr6 & 2.4.1.131 & Saccharomyces cerevisiae & P54070 \\
\hline Mannosyltransferase Ktr7 & 2.4.1.131 & Saccharomyces cerevisiae & P40504 \\
\hline Mannosyltransferase Yur1 & 2.4.1.131 & Saccharomyces cerevisiae & P26725 \\
\hline \multicolumn{4}{|l|}{ Family 16 (inverting) } \\
\hline$\beta$-1,2-GIcNAc transferase ॥ & 2.4.1.143 & Human & Q10469 \\
\hline$\beta-1,2-G \mid c N A c$ transferase II & 2.4.1.143 & Rat & Q09326 \\
\hline \multicolumn{4}{|l|}{ Family 19} \\
\hline Lipid A disaccharide synthase $L p x B$ & 2.4.1.182 & Escherichia coli & P10441 \\
\hline Lipid A disaccharide synthase $L p x B$ & 2.4.1.182 & Haemophilus influenzae & P45011 \\
\hline LpXB protein & & Proteus mirabilis & Y09263 \\
\hline LpxB protein & & Synechocystis sp. & D64000 \\
\hline \multicolumn{4}{|l|}{ Family 20 (retaining) } \\
\hline$\alpha, \alpha$-Trehalose-phosphate synthase & 2.4.1.15 & Arabidopsis thaliana & Y08568 \\
\hline$\alpha, \alpha$-Trehalose-phosphate synthase Tps1 & 2.4.1.15 & Aspergillus niger & U07184 \\
\hline$\alpha, \alpha$-Trehalose-phosphate synthase TpsB & 2.4.1.15 & Aspergillus niger & U63416 \\
\hline Unknown ZK54.2 & & Caenorhabditis elegans & U58737 \\
\hline$\alpha, \alpha$-Trehalose-phosphate synthase Tps1 & 2.4.1.15 & Candida albicans & Y07918 \\
\hline$\alpha, \alpha$-Trehalose-phosphate synthase OtsA & 2.4.1.15 & Escherichia coli & P31677 \\
\hline$\alpha, \alpha$-Trehalose-phosphate synthase Tps 1 & 2.4.1.15 & Kluyveromyces lactis & Q07158 \\
\hline OtsA protein & & Mycobacterium leprae & U15187 \\
\hline$\alpha, \alpha$-Trehalose-phosphate synthase Tps1 & 2.4.1.15 & Saccharomyces cerevisiae & Q00764 \\
\hline$\alpha, \alpha$-Trehalose-phosphate synthase Tps2 & 2.4.1.15 & Saccharomyces cerevisiae & P31688 \\
\hline$\alpha, \alpha$-Trehalose-phosphate synthase Tps3 & 2.4.1.15 & Saccharomyces cerevisiae & P38426 \\
\hline$\alpha, \alpha$-Trehalose-phosphate synthase Tps 1 & 2.4.1.15 & Schizosaccharomyces pombe & P40387 \\
\hline OtsA protein & & Synechocystis sp. & D90913 \\
\hline \multicolumn{4}{|l|}{ Family 21 (retaining) } \\
\hline Unknown F20B4.6 & & Caenorhabditis elegans & U58735 \\
\hline Unknown T06C12.C & & Caenorhabditis elegans & Z81116 \\
\hline Unknown YK29C8.5 & & Caenorhabditis elegans & U53332 \\
\hline Ceramide glucosyltransferase & 2.4.1.80 & Human & Q16739 \\
\hline Unknown (ORF SLR0813) & & Synechocystis sp. & D90911 \\
\hline
\end{tabular}


Table 1 (cont.)

\begin{tabular}{|c|c|c|c|}
\hline Description ${ }^{(a)}$ & EC number ${ }^{(b)}$ & Organism & Accession no. ${ }^{(c)}$ \\
\hline \multicolumn{4}{|l|}{ Family 22} \\
\hline Unknown C14A4.3 & & Caenorhabditis elegans & Z49909 \\
\hline Mannosyltransferase & & Human & D42138 \\
\hline Mannosyltransferase & & Saccharomyces cerevisiae & X96417 \\
\hline \multicolumn{4}{|l|}{ Family 23 (inverting) } \\
\hline Fucosyltransferase (NodZ) & & Azorhizobium caulinodans & L18897 \\
\hline Fucosyltransferase (NodZ) & & Bradyrhizobium japonicum & L22756 \\
\hline$N$-Acetyl- $\beta$-D-glucosaminide $\alpha$-1,6-fucosyltransferase & & Pig & D86723 \\
\hline \multicolumn{4}{|l|}{ Family 24} \\
\hline Unknown C12C8.D & & Caenorhabditis elegans & $Z 81467$ \\
\hline UDP-glucose glycoprotein glucosyltransferase & & Drosophila melanogaster & U20554 \\
\hline Killer toxin-resistance protein Kre5 & & Saccharomyces cerevisiae & P22023 \\
\hline UDP-glucose glycoprotein glucosyltransferase & & Schizosaccharomyces pombe & U38417 \\
\hline \multicolumn{4}{|l|}{ Family 25} \\
\hline Unknown D2045.9 & & Caenorhabditis elegans & Z35639 \\
\hline LbgA protein & & Haemophilus ducreyi & U58147 \\
\hline Lic2B protein & & Haemophilus influenzae & U36398 \\
\hline LPS biosynthesis protein & & Haemophilus influenzae & $\times 56903$ \\
\hline LPS biosynthesis protein & & Haemophilus influenzae & L19441 \\
\hline LPS biosynthesis protein Lex-1 & & Haemophilus influenzae & U32736 \\
\hline Lex2B protein & & Haemophilus influenzae & U05670 \\
\hline Galactosyltransferase LgtB & & Neisseria gonorrhoeae & U14554 \\
\hline Galactosyltransferase LgtE & & Neisseria gonorrhoeae & U14554 \\
\hline Glycosyltransferase LgtB & & Neisseria meningitidis & U25839 \\
\hline Glycosyltransferase LgtE & & Neisseria meningitidis & U25839 \\
\hline LpsA protein & & Pasteurella haemolytica & U15958 \\
\hline \multicolumn{4}{|l|}{ Family 26} \\
\hline AceB protein & & Acetobacter xylinum & X94981 \\
\hline UDP-N-acetylmannosaminuronic acid transferase & & Escherichia coli & P27836 \\
\hline Teichoic acid biosynthesis protein A & & Escherichia coli & P27620 \\
\hline UDP-N-acetylmannosaminuronic acid transferase & & Salmonella typhimurium & P37457 \\
\hline Cps19fF protein & & Streptococcus pneumoniae & U09239 \\
\hline Unknown (ORF SLR1118) & & Synechocystis sp. & D90899 \\
\hline Unknown (ORF SLR1271) & & Synechocystis sp. & D90913 \\
\hline GumM protein & & Xanthomonas campestris & U22511 \\
\hline
\end{tabular}

described by the present work could provide an aid to structural interpretation and, when more structures become available, suggest possible search models for molecular replacement.

For those families where both the NDP-sugar and the linkage formed are known, i.e., all families except families 19, 22, 24, 25 and 26, the classification based on sequence similarity consistently differentiates retaining from inverting enzymes. This is consistent with the conservation of the catalytic machinery of these enzymes within each family. Almost half of the classified sequences have unknown or uncertain functions (Table 1). A fundamental basis for a classification must be that it has predictive power. The present classification allows the prediction of their global function (i.e. NDP-glycosyltransferase) and product stereochemistry (inverted or retained anomeric configuration).

By analogy to glycoside hydrolases, the catalytic machinery of glycosyltransferases is likely to involve Asp and/or Glu residues whose side chains have the appropriate reactivity to act as the general base for acceptor activation or as the nucleophile for the formation of a glycosyl-enzyme intermediate. Site-directed mutagenesis of ribosyltransferases has shown that specific Glu residues are essential for glycosyltransferase activity [20]. For each family of glycosyltransferases, the list of the invariant Asp or Glu residues is therefore likely to contain catalytic residues. In some of the families we describe there are so few such conserved residues that the catalytic machinery is probably directly identi- fiable. Examples include families 1, 9 and 11 with one invariant Asp, families 5 and 25 with one invariant Glu, families 3 and 4 with two invariant Glu and families 2, 8, 9 and 20 with two invariant Asp residues.

The EC recommendations place all hexosyltransferases in the same subclass (EC 2.4.1.x), regardless of the sugar donor used by the enzymes. There are clear structural, evolutionary and mechanistic similarities between several glycosyltransferases using glycosides as activated sugar donors and glycoside hydrolases. For example, cyclodextrin glucanotransferases (EC 2.4.1.19) and starch branching enzymes (EC 2.4.1.18) are clearly related to a large number of starch-hydrolysing enzymes forming family 13 of glycoside hydrolases [4,6,7,21]. Similarly, endo-xyloglucan transferases (EC 2.4.1.207) display significant similarities to glycoside hydrolase family 16 members [7]. In contrast, we have been unable to detect any sequence similarity between the NDPsugar glycosyltransferases we have analysed and glycoside hydrolases. This probably reflects particular constraints on the active site of these glycosyltransferases which must accommodate the bulky NDP-moiety.

On several occasions, we observed that enzymes acting on similar substrates with the same mechanism, and classified in different families, displayed intriguing local similarities which could not be extended to the rest of the sequence. This situation, which perhaps reflects the limitations of sequence comparisons at 
very high divergence, is reminiscent of the grouping of glycoside hydrolase families into clans where the only sequence similarity is found around the catalytic machinery [22]. An example of such possibly related families are families 3, 4 and 5, which display limited local similarities. Similarly, families 11 and 23 could perhaps be grouped based on the specific instance of the motif VHVRRTD in a family 23 enzyme (porcine $N$-acetyl- $\beta$-Dglucosaminide $\alpha$-1,6-fucosyltransferase) which is almost identical with one of the three highly conserved motifs in family 11 (VHVRRGD motif). Conversely, the proposed grouping of $\alpha$ 1,3-fucosyltransferases and $\alpha$-1,2-fucosyltransferases [23] cannot be confirmed as the corresponding families (10 and 11) do not bear even one conserved residue. Only structural resolution will allow the reliable grouping of families into 'superfamilies' or 'clans'.

That there are several polyspecific families leads to the proposition that the observed differences in substrate specificity probably reflect divergent evolution from an ancestral form of glycosyltransferase. Conversely, we have identified at least one example of an enzyme activity (lipopolysaccharide 1,2- $N$-acetylglucosaminyltransferase; EC 2.4.1.56) which appears in two distinct families (4 and 9), suggesting that this could constitute an example of convergent evolution.

Genome sequencing projects are increasingly delivering large numbers of potential glycosyltransferase sequences and the present classification that brings together structural, mechanistic and sequence-based information is clearly of biocomputing importance. Significantly, a possible function was recently proposed for secreted Fringe-like signalling molecules based on distant sequence similarity with glycosyltransferases sequences [24]. It is our intention to set up an electronic access to this classification similar to that already implemented for the glycosidases [7].

J. C. thanks the Australian Government Department of Industry, Science and Tourism for financial support of his visit to CERMAV. J.C. also thanks the Sugar Research and Development Corporation for partial funding of this research. G.J.D. is a Royal Society University Research Fellow.

James A. CAMPBELL*, Gideon J. DAVIES $\uparrow$, Vincent BULONE $\$ S$ and Bernard HENRISSAT $\$$

*CSIRO Tropical Agriculture, 306 Carmody Road, St. Lucia, Q 4067, Australia, †Department of Chemistry, University of York, Heslington, York Y01 5DD, U.K., and \$Centre de Recherches sur les Macromolécules Végétales (Affiliated with the Joseph Fourier University), CNRS, BP 53, F-38041 Grenoble Cedex 9, France

$\S$ To whom correspondence should be addressed.

1 Kleene, R. and Berger, E. G. (1993) Biochim. Biophys. Acta 1154, 283-325

2 Sinnott, M. L. (1990) Chem. Rev. 90, 1171-1202

3 International Union of Biochemistry and Molecular Biology (1992) Enzyme Nomenclature: Recommendations of the Nomenclature Committee of the International Union of Biochemistry and Molecular Biology, Academic Press, San Diego

4 Henrissat, B. (1991) Biochem. J. 280, 309-316

5 Rawlings, N. D. and Barrett, A. J. (1993) Biochem. J. 290, 205-218

6 Henrissat, B. and Bairoch, A. (1993) Biochem. J. 293, 781-788

7 Henrissat, B. and Bairoch, A. (1996) Biochem. J. 316, 695-696

8 Rawlings, N. D. and Barrett, A. J. (1994) Methods Enzymol. 244, 1-15

9 Rawlings, N. D. and Barrett, A. J. (1995) Methods Enzymol. 248, 105-120

10 Davies, G. and Henrissat, B. (1995) Structure 3, 853-859

11 Gebler, J., Gilkes, N. R., Claeyssens, M., Wilson, D. B., Béguin, P., Wakarchuk, W. W., Kilburn, D. G., Miller, Jr., R. C., Warren, R. A. J. and Withers, S. G. (1992) J. Biol. Chem. 267, 12559-12561

12 Geremia, R. A., Petroni, A., lelpi, L. and Henrissat, B. (1996) Biochem. J. 318, $133-138$

13 Saxena, I., Brown, Jr., R. M., Fèvre, M., Geremia, R. A. and Henrissat, B. (1995) J. Bacteriol. 177, 1419-1424

14 Altschul, S. F., Gish, W., Miller, W., Myers, E. W. and Lipman, D. J. (1990) J. Mol. Biol. 215, 403-410
15 Durand, P., Canard, L. and Mornon, J. P. (1997) Comput. Appl. Biosci., in the press

16 Gaboriaud, C., Bissery, V., Benchetrit, T. and Mornon, J. P. (1987) FEBS Lett. 224, $149-155$

17 Lemesle-Varloot, L., Henrissat, B., Gaboriaud, C., Bissery, V., Morgat, A. and Mornon, J. P. (1990) Biochimie 72, 555-574

18 Chothia, C. and Lesk, A. M. (1986) EMBO J. 5, 823-826

19 Vrielink, A., Rüger, W., Driessen, H. P. C. and Freemont, P. S. (1994) EMBO J. 13 3413-3422

20 Takada, T., lida, K. and Moss, J. (1995) J. Biol. Chem. 270, 541-544

21 Jespersen, H. M., MacGregor, E. A., Henrissat, B., Sierks, M. R. and Svensson, B. (1993) J. Protein Chem. 12, 791-805

22 Henrissat, B., Callebaut, I., Fabrega, S., Lehn, P., Mornon, J. P. and Davies, G. (1995) Proc. Natl. Acad. Sci. U.S.A. 92, 7090-7094

23 Breton, C., Oriol, R. and Imberty, A. (1996) Glycobiology 6, vii-xii

24 Yuan, Y. P., Schultz, J., Mlodzik, M. and Bork, P. (1997) Cell 88, 9-11

Received 23 April 1997

\section{The Factor I and follistatin domain families: the return of a prodigal son}

The Factor I/membrane-attack complex (FIMAC) domain has been identified in three complement proteins of immune defence and was believed to be unique [1]. It is present in the essential regulatory protease Factor $\mathrm{I}(\mathrm{FI})$ that is specific for the complement proteins $\mathrm{C} 3 \mathrm{~b}$ and $\mathrm{C} 4 \mathrm{~b}$ when complexed with their cofactors [2], and also in the terminal components $\mathrm{C} 6$ and $\mathrm{C} 7$ of the membrane-attack complex [3,4]. FI contains an N-terminal FIMAC domain (67 residues), a CD5 or scavenger-receptor cysteine-rich domain, two low-density-lipoprotein-receptor domains, and a serine-protease domain. Both $\mathrm{C} 6$ and $\mathrm{C} 7$ contain two C-terminal FIMAC domains. Here we show that database searches based on the FIMAC sequence indicated similarities with the follistatin (FS) sequence family (Figure 1). Similarities were also found in the predicted secondary structures of the FIMAC and FS domains (Figure 2). We therefore propose that the FIMAC domain is a distant member of the larger FS superfamily [5,6]. For FI, all the domain types in its structure have now been identified as members of larger cellular-receptor or extracellular-matrix domain superfamilies [7-9]. The FS superfamily of the extracellular matrix and the endocrine system now includes the immune equivalent of this domain.

Seven FIMAC sequences [3,4,10-12] were used in database searches of more than 59000 SWISSPROT sequences using BLITZ which is based on the program MPsrch with the BLOSUM62 amino acid substitution matrix [13]. Table 1 indicates hits with agrin in the FS family with 19-24\% sequence matches. Lower matches in the top 50 hits (Table 1) included

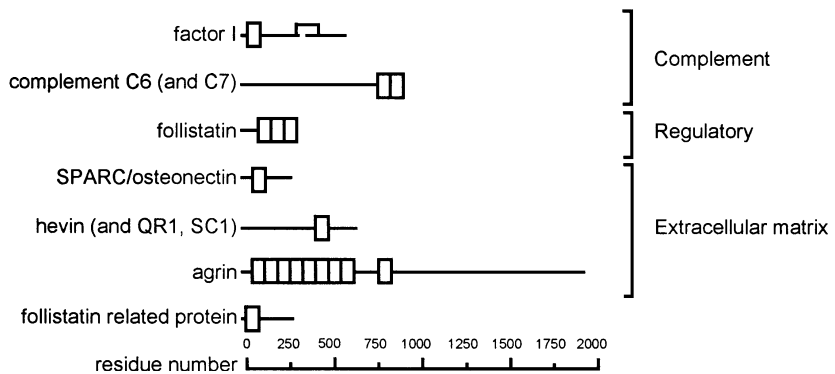

Figure 1 Occurrence of FIMAC and FS domains in the sequences of plasma, regulatory and extracellular matrix proteins

Each FIMAC or FS domain is denoted by a box. 


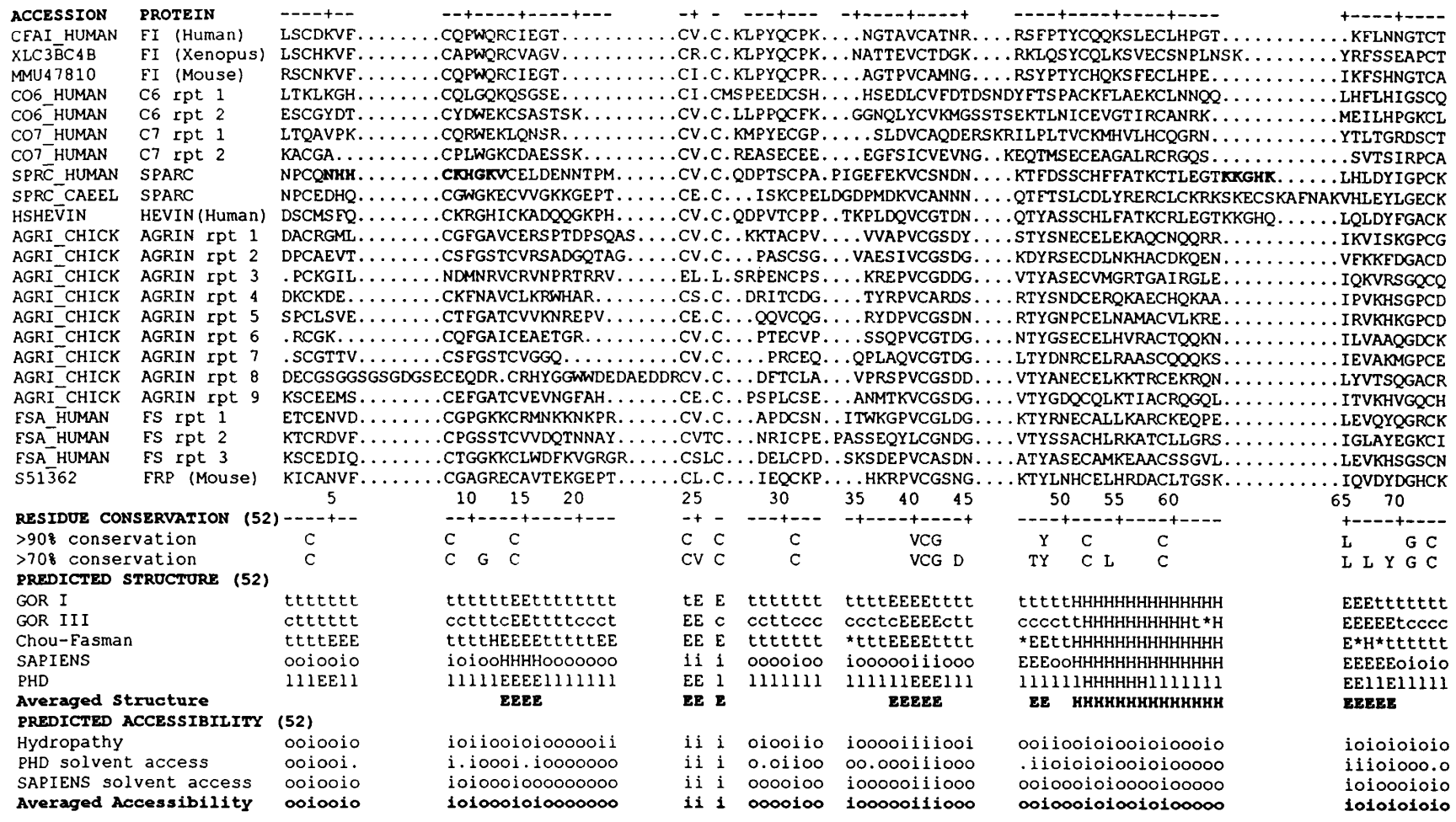

\section{Figure 2 Summary of the multiple sequence alignment of FIMAC/FS sequences}

A total of 52 was used in the full alignment. The 29 not shown in the Figure have SWISSPROT accession codes SPRC_BOVIN, SPRC_MOUSE, SPRC_RAT, SPRC_CHICK, SPRC_XENLA, QR1_COTJA, SC1_RAT, DYGAGR, AGRI_RAT, FSA_PIG, FSA_RAT, FSA_SHEEP, FSA_XENLA, from which the full alignment is readily reconstructed. The two $\mathrm{Cu}^{2+}$-binding regions of the SPARC sequences are in bold. The alignment yielded a consensus length of 74 residues which is conserved in over $50 \%$ of the 52 sequences. The most commonly occurring residues that show $90 \%$ and $70 \%$ conservation within a given subtype $(I=V=L=M ; D=E ; R=K=H ; F=Y=W=H ; G=A=S)$ are indicated. The averaged predicted structures are based on the presence of at least $2 / 5$ secondary structures or 2/3 accessibility states at each residue position. Abbreviations are as follows; $\mathrm{H}, \alpha$-helix; $\mathrm{E}, \beta$-sheet; I, $\mathrm{C}$ and $\mathrm{t}$, loop, coil and turn; 0 , solvent-accessible; i, solvent-inaccessible.

Table 1 BLITZ database search of more than 59000 sequences

\begin{tabular}{lllr}
\hline $\begin{array}{l}\text { Probe FIMAC sequence } \\
\text { (see Figure 2) }\end{array}$ & $\begin{array}{l}\text { FS sequences detected } \\
(P \text { better than 10-4) }\end{array}$ & Probability $(P)$ & $\begin{array}{l}\text { Total of FS sequences } \\
\text { in top 50 positions }\end{array}$ \\
\hline CFAI_HUMAN (residues 41-107) & AGRI_RAT & $4 \times 10^{-5}$ & 10 \\
XLC3BC4B (residues 39-101) & AGRI_RAT & $4 \times 10^{-6}$ & 9 \\
MMU47810 (residues 44-110) & AGRI_CHICK & $3 \times 10^{-7}$ & 16 \\
& AGRI_RAT & $9 \times 10^{-7}$ & 17 \\
CO6_HUMAN (residues 766-837) & None & $7 \times 10^{-8}$ & 16 \\
CO7_HUMAN (residues 771-839) & AGRI_CHICK & $9 \times 10^{-6}$ & \\
\hline
\end{tabular}

other agrin repeats, FS, SPARC (secreted protein, acidic, rich in cysteine; also known as osteonectin) and the SPARC homologues QR1 and SC1, all of which belong to the FS family. The BLITZ matches with the FS domains of chicken and rat agrin extended over 52-65 residues of the probe FIMAC sequences (Table 1) and aligned eight to ten of the eight or ten cysteine residues in the FIMAC sequences. Another database search using BLASTP [14] to scan more than 71000 sequences showed that AGRI_RAT and AGRI_CHICK were scored with statistically significant probabilities $(P)$ of $10^{-5}-10^{-6}$ by FIMAC sequences from $\mathrm{FI}$ and C7. Likewise version 3 of FASTA [15] yielded similar results with more than 59000 sequences, in which FS domains in agrin, QR1 and FS also scored highly with probabilities $E(59000)$ less than 0.05 , and lower matches included other FS domains. FASTA matched eight to ten of the eight or ten cysteine residues of FIMAC with their equivalents in the FS domain. Blockmaker
[16], with the FIMAC sequences and the FS domains from agrin and SPARC (Figure 2), produced two blocks, one of which aligned with both the FIMAC and FS sequences. Likewise, MACAW [17] generated a 28-residue block of ten aligned sequences from the top matches in the BLITZ search, which corresponded to that identified by Blockmaker [16] (residues 31-60 in Figure 2). Whereas the 28-residue block lacked the C6 or C7 FIMAC sequences, its occurrence was statistically significant.

Since these analyses showed a relationship between the FIMAC and FS sequences, a combined alignment was constructed using 52 sequences (Figure 2). The consensus length is 74 residues with ten conserved cysteine residues. These cysteine residues are assumed to be bridged. Although the disulphide pairings are unknown, cysteine mutations in the first FIMAC of C6 and C7 suggest that $\mathrm{Cys}^{3}-\mathrm{Cys}^{14}$ are paired (the first and third Cys in 
Figure 2). $\mathrm{Cys}^{8}, \mathrm{Cys}^{24}$, $\mathrm{Cys}^{26}$ and $\mathrm{Cys}^{59}$ (the second, fourth, fifth and ninth Cys in Figure 2) are missing in agrin domain 3 and may form two pairs if the domain structure is unaltered. Whereas C-terminal sequence similarities with the disulphide-rich Kazaltype inhibitors of the ovomucoid family and N-terminal similarities with the epidermal-growth-factor family have been noted $[6,8]$, the proposed disulphide pairings in the alignment are mutually exclusive in the two families [5]. Figure 2 also shows conserved buried hydrophobic residues. These are attributable to the packing of the protein core, and most are present in both the FIMAC and FS sequences.

The FIMAC and FS sequences were also compared by computing consensus secondary-structure predictions from the alignment [18,19]. Use of the GORI, GORIII, Chou Fasman, PHD and SAPIENS prediction algorithms gave an averaged $\beta \beta \beta \beta \alpha \beta$ structure with five $\beta$-strands and one $\alpha$-helix (Figure 2). Residues 38-74 resemble the Kazal-type inhibitors. Interestingly, they resulted in a $\beta \beta \alpha \beta$ prediction that is very similar to the observed $\beta \beta \alpha \beta$ secondary structure in ovomucoid when analysed using DSSP (Brookhaven database codes: 1ovo-4ovo). Cys ${ }^{35}$ and $\mathrm{Cys}^{38}$ are located on the $\alpha$-helix of ovomucoid and correlate well with $\mathrm{Cys}^{52}$ and $\mathrm{Cys}^{59}$ in FIMAC/FS, which are located on the $\alpha$-helix predicted between residues 51 and 64 (Figure 2). Cys ${ }^{52}$ and $\mathrm{Cys}^{59}$ in FIMAC/FS will be positioned on the same side of this $\alpha$-helix as $\mathrm{Cys}^{35}$ and $\mathrm{Cys}^{38}$ in ovomucoid, since $\mathrm{Cys}^{52}$ and Cys $^{59}$ will be separated by an extra turn of the predicted $\alpha$-helix in the FIMAC/FS domain compared with ovomucoid. The presence of the extra N-terminal residues in FIMAC before the region of similarity with ovomucoid implies that a structural relationship to ovomucoid is possible, but this will be modified. Figure 2 also shows large sequence insertions in the alignment, and these occur in regions predicted to be surface loops as desired. The loop between residues 64 and 65 corresponds to the $\mathrm{Cu}^{2+}$-binding region of the SPARC proteins [5] that is implicated in cellular proliferation, but is absent from other FIMAC and FS domains. More importantly, application of averaged secondarystructure predictions to each of the FIMAC and FS sequence families yielded results that were very similar to the predicted $\beta \beta \beta \beta \alpha \beta$ structure for all 52 sequences and support the proposed identity between the two families.

The phylogenic relationship between the FIMAC and FS sequences was investigated using PHYLIP [20]. An unrooted tree showed that the FIMAC sequences occupied a separate branch from the FS sequences. The lengths of the exons in SPARC, agrin, FI, C6 and C7 are in agreement with Figure 2, whereas their boundaries are not conserved. In murine SPARC, the FS domain is encoded by exons 5 and 6 with intron boundaries of class 1-1 [5], and correlates well with the FS domains in agrin, which are encoded by one or two exons and have intron boundaries of class 1-1 [21]. In human FI, the FIMAC domain is within exon 2, but with intron boundaries of class 0-1 [22]. The two FIMAC domains in human $\mathrm{C} 6$ and $\mathrm{C} 7$ are encoded across exons 15,16 and 17 with intron boundaries of class 1-2, 2-1 and 1 -undefined respectively $[3,4]$. The lack of conserved intron/exon structure is similar to that found in the serine-protease domain [22].

The relationship between the FIMAC and FS families is matched functionally in that all proteins containing FIMAC and FS domains are extracellular and participate in protein-protein interactions (Figure 1). The FS domain has been identified in extracellular matrix proteins that modulate cell-matrix interactions (SPARC), induce aggregation of nicotinic acetylcholine receptors (agrin) and in ovaries and the pituitary that bind cytokines (FS) [5,6]. Hevin is isolated from high endothelial venules of tonsils which allow high levels of lymphocyte extra- vasion from blood and may facilitate this lymphocyte migration [23]. SPARC is released by platelet degranulation, is synthesized by fibroblasts and macrophages at sites of wound repair, and may regulate deposition or assembly of extracellular matrix proteins. The specificity of the FS domain for its ligand is indicated by SPARC and FS. SPARC binds to platelet-derived growth factor, albumin, thrombospondin and various collagen types. FS binds to activin and inhibin, which are transforminggrowth-factor- $\beta$-like cytokines. Despite the sequence similarity to ovomucoid, no protease-inhibitory activity has been reported to date for FS-containing proteins [6]. FI interacts with the complement components $\mathrm{C} 3 \mathrm{~b}$ and $\mathrm{C} 4 \mathrm{~b}$, whereas $\mathrm{C} 6$ and $\mathrm{C} 7$ interact with complement component $\mathrm{C} 5 \mathrm{~b}$ during formation of the membrane-attack complex. As $\mathrm{C} 3 \mathrm{~b}, \mathrm{C} 4 \mathrm{~b}$ and $\mathrm{C} 5 \mathrm{~b}$ are all related in sequence, it will be of interest to determine whether all three contain a similar target fold for FIMAC. Given the relationship to ovomucoid, it will be of interest to determine whether FIMAC in FI can inhibit its own serine-protease domain. From X-ray and neutron-scattering analyses of FI, one model that is consistent with the data is a semi-compact V-shaped structure, the dimensions of which place these two domains proximate to each other [24].

We thank the Wellcome Trust for grant support and Dr. R. B. Sim for useful discussions.

\section{Christopher G. ULLMAN and Stephen J. PERKINS}

Department of Biochemistry and Molecular Biology, Royal Free Hospital School of Medicine, Rowland Hill Street, London NW3 2PF, U.K.

1 To whom correspondence and requests for offprints should be addressed.

1 Law, S. K. A. and Reid, K. B. M. (1995) Complement, 2nd edn., IRL Press, Oxford

2 Sim, R. B., Day, A. J., Moffatt, B. E. and Fontaine, M. (1993) Methods Enzymol. 223, 13-35

3 Hobart, M. J., Fernie, B. and DiScipio, R. G. (1993) Biochemistry 32, 6198-6205

4 Hobart, M. J., Fernie, B. A. and DiScipio, R. G. (1995) J. Immunol. 154, 5188-5194

5 Lane, T. F. and Sage, E. H. (1994) FASEB J. 8, 163-173

6 Patthy, L. and Nikolics, K. (1993) Trends Neurosci. 16, 76-81

7 Perkins, S. J. and Smith, K. F. (1993) Biochem. J. 295, 109-114

8 Moestrup, S. K. (1994) Biochim. Biophys. Acta 1197, 197-213

9 Resnick, D., Pearson, A. and Krieger, M. (1994) Trends Biochem. Sci. 19, 5-8

10 Catterall, C. F., Lyons, A., Sim, R. B., Day, A. J. and Harris, T. J. (1987) Biochem. J. 242, 849-856

11 Kunnath-Muglia, L. M., Chang, G. H., Sim, R. B., Day, A. J. and Ezekowitz, R. A. (1993) Mol. Immunol. 30, 1249-1256

12 Minta, J. O., Wong, M. J., Kozak, C. A., Kunnath-Muglia, L. M. and Goldberger, G. (1996) Mol. Immunol. 33, 101-112

13 Sturrock, S. S. and Collins, J. F. (1993) MPsrch, Version 1.5, Biocomputing Research Unit, University of Edinburgh

14 Altschul, S. F., Warren, G., Miller, W., Myers, E. W. and Lipman, D. J. (1990) J. Mol. Biol. 215, 403-410

15 Pearson, W. R. and Lipman, D. J. (1988) Proc. Natl. Acad. Sci. U.S.A. 85, 2444-2448

16 Henikoff, S., Henikoff, J. G., Alford, W. J. and Pietrokovski, S. (1995) Gene 163, GC (Gene-COMBIS) 17-26

17 Lawrence, C. E., Altschul, S. F., Boguski, M. S., Liu, J. S., Neuwald, A. F. and Wootton, J. C. (1993) Science 262, 208-214

18 Edwards, Y. J. K. and Perkins, S. J. (1996) J. Mol. Biol. 260, 277-285

19 Brissett, N. C. and Perkins, S. J. (1996) FEBS Lett. 388, 211-216

20 Felsenstein, J. (1989) Cladistics 5, 164-166

21 Rupp, F., Özçelik, T., Linial, M., Peterson, K., Francke, U. and Scheller, R. (1992) J. Neurosci. 12, 3535-3544

22 Vyse, T. J., Bates, G. P., Walport, M. J. and Morley, B. J. (1994) Genomics 24 $90-98$

23 Girard, J. P. and Springer, T. A. (1995) Immunity 2, 113-123

24 Perkins, S. J., Smith, K. F. and Sim, R. B. (1993) Biochem. J. 295, 101-108

Received 6 May 1997 